\title{
Au cœur de Coriolan : la démocratie en questions
}

\section{Gérald Garutti}

\section{(2) OpenEdition \\ Journals}

\section{Édition électronique}

URL : http://journals.openedition.org/shakespeare/1613

DOI : 10.4000/shakespeare.1613

ISSN : 2271-6424

Éditeur

Société Française Shakespeare

Édition imprimée

Date de publication : 1 mars 2011

Pagination : 95-104

ISBN : 2-9521475-7-4

Référence électronique

Gérald Garutti, «Au cœur de Coriolan : la démocratie en questions », Actes des congrès de la Société française Shakespeare [En ligne], 28 | 2011, mis en ligne le 15 février 2011, consulté le 02 mai 2019. URL : http://journals.openedition.org/shakespeare/1613; DOI : 10.4000/shakespeare.1613 


\section{Shakespeare et la Cité}

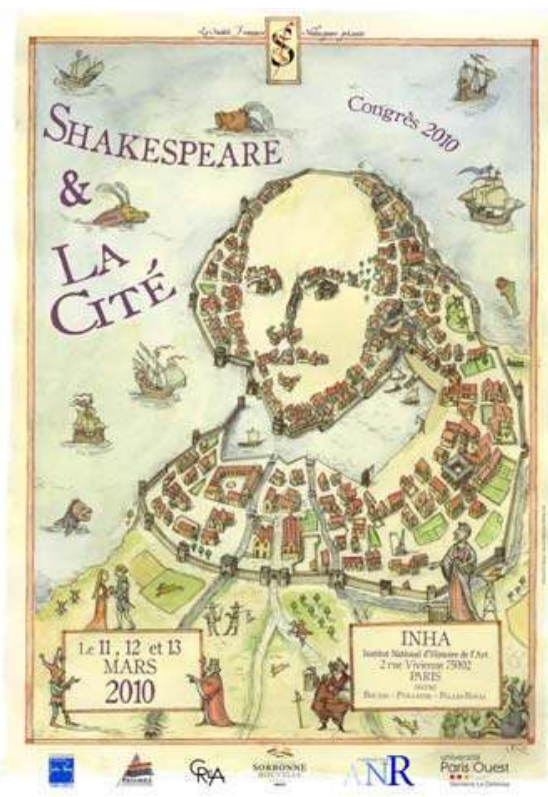

actes du Congrès

organisé par la

SOCIÉTÉ FRANÇAISE SHAKESPEARE

les 11,12 et 13 mars 10

textes réunis par

Pierre KAPITANIAK

sous la direction de

Dominique GoY-BLANQUET 
COUVERTURE :

Edouard Lekston 2010

conception graphique et logo

Pierre Kapitaniak

\section{(C) 2011 Société Française Shakespeare Institut du Monde Anglophone} Université de Paris III - Sorbonne Nouvelle 5 rue de l'École de Médecine 75006 Paris

www.societefrancaiseshakespeare.org réservés pour tous les pays 


\title{
Au CEUR de Coriolan : LA DÉMOCRATIE EN QUESTIONS
}

\author{
Gérald GARUTTI
}

Comment vivre ensemble malgré les différences et les différends ? La démocratie est-elle le pire des régimes à l'exception de tous les autres? N'avons-nous le choix qu'entre la démagogie des tribuns et la tyrannie des hommes forts ? Faut-il préférer la sécurité à la liberté, et l'ordre à la justice? Autant de problèmes abordés par Shakespeare dans Coriolan. Rome y oscille entre trois régimes décadents : une démocratie rêvée qui vire à la démagogie, une aristocratie effective mâtinée d'oligarchie militaire, une pente tyrannique qui rappelle la monarchie exécrée. Dans sa démesure élitiste et son refus de jouer la comédie du pouvoir démocratique, le héros s'avère incapable de convertir son triomphe militaire en suprématie politique - ignorant que la politique est la continuation de la guerre par d'autres moyens. Ainsi cette tragédie brasse-t-elle des enjeux d'une déchirante actualité pour nos démocraties en souffrance : guerre civile latente, lutte des classes exacerbée, crise perpétuelle, instabilité permanente, bestiaire fratricide, dissension infinie, impossible concorde civile, corruption fatale, salut par l'impérialisme, expulsion du héros, bureaucratie désenchantée, et règne ultime de la représentation.

How can we live together despite our differences and contradictions? Is democracy the worst of regimes all others excepted? Can we but choose between tribunes' demagogy and strong men's tyranny? Must we prefer safety to freedom, and order to justice? All these issues are addressed by Shakespeare in Coriolanus. There, Rome oscillates between three decadent regimes: a dreamed democracy that turns to demagogy, an effective aristocracy tainted with military oligarchy, and a tyrannical slope reminiscent of loathed monarchy. In his elitis excess and his refusal to act the comedy of democratic power, the hero proves unable to transform his military triumph into political supremacy - unaware that politics is the continuation of war by other means. Thus this tragedy tackles issues that are poignantly topical for our suffering democracies: latent civil war, exacerbated class struggle, perpetual crisis, permanent instability, fratricidal bestiary, infinite dissension, impossible civil concord, fatal corruption, salvation through imperialism, expulsion of the hero, disenchanted bureaucracy, and ultimate reign of representation.

\section{Vertige politique}

$\mathrm{C}$ omment vivre ensemble quand on est différents? La démocratie est-elle le pire des régimes à l'exception de tous les autres? N'avons-nous le choix qu'entre la démagogie des tribuns et la tyrannie des « hommes forts » ? Faut-il préférer la sécurité à la liberté, et à la révolution sociale, l'ordre autoritaire, fût-il présenté comme «juste»? Autant de problèmes brillamment abordés par Shakespeare dans son ultime tragédie, la plus complexe et la plus éminemment politique, Coriolan. Située dans la Rome républicaine balbutiante (-488), écrite dans l'Angleterre moderne naissante (1607), elle expose des enjeux d'une déchirante actualité pour nos républiques et démocraties en souffrance. À cet égard, trois problématiques soustendent la pièce: le meilleur régime, la lutte des classes et 
l'impérialisme colonial. Chaque étape articule ces tensions sans prétendre les résoudre.

D'emblée, un spectre hante Rome : le spectre de la guerre civile. La Ville est plus qu'en ébullition, elle est en guerre(s). Non seulement contre son ennemi et voisin, le peuple volsque, mais surtout contre elle-même. Dès la première scène, tout est possible. En équilibre instable, la jeune république romaine vacille sur ses fondements encore mal assurés. Le monde va peut-être changer de base - mais pour quel destin ? Défilent ainsi de nombreuses options politiques concurrentes, en une dialectique de l'ordre et du chaos : révolte populaire, révolution sociale, compromis réformiste, paternalisme conservateur, répression militaire, représentation démocratique, union sacrée face à l'invasion volsque...

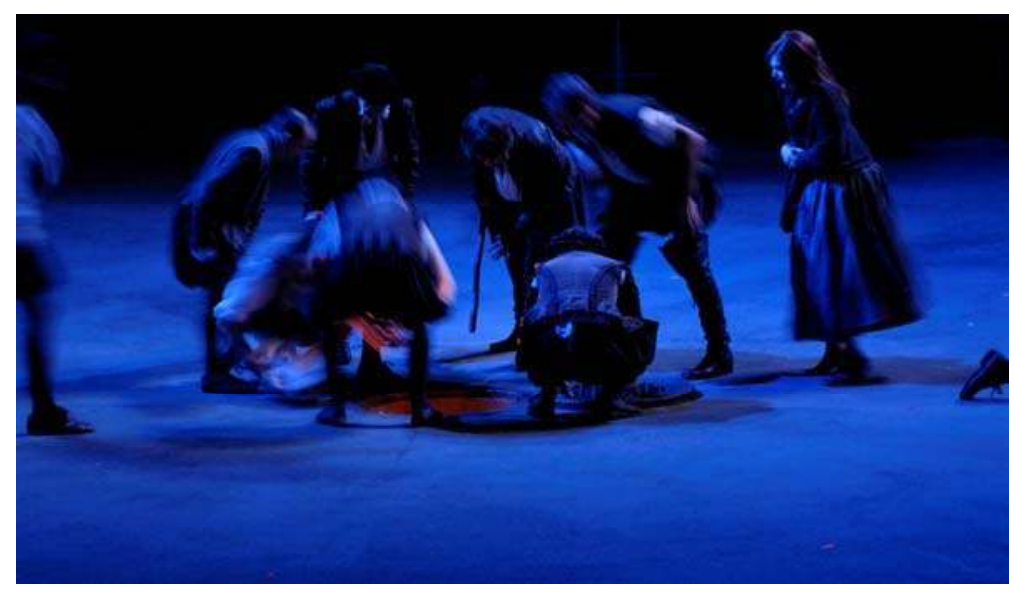

\section{Deux classes}

Ce vertige politique exprime d'abord la radicalité de la lutte des classes à Rome. Le monde s'y divise en deux grandes catégories sociales, la classe dominante des patriciens tout puissants, et la classe dominée des plébéiens sans droits, ni richesses, ni pouvoirs. Parfois, par son urgence, la guerre extérieure occulte un temps cette ligne de fractures intestines. Mais, pendant deux siècles et demi (-509/-264), c'est-à-dire au moins jusqu'aux Guerres Puniques livrées contre cette grande rivale méditerranéenne qu'est la civilisation carthaginoise, s'impose comme 
combat structurel l'émancipation des classes populaires à travers l'accès des plébéiens aux droits fondamentaux, l'avènement d'une représentation politique populaire, le rééquilibrage démocratique des institutions et la conquête d'une moindre injustice sociale. À cette poussée démocratique relative, l'aristocratie résiste en dénonçant les dangers de cette hydre aux mille têtes.

Auscultant une République d'à peine vingt ans encore à la croisée des chemins, la tragédie de Shakespeare croise la question ontologique du meilleur régime, question classique déployée de Platon à Montesquieu, avec la double interrogation moderne, pragmatique, formulée sans ambages par Machiavel: 1. Comment prendre le pouvoir ? 2. Comment le conserver ? Problème de la prise du pouvoir que se pose tout prince potentiellement tyrannique, mais également tout révolutionnaire, comme s'y attellera Lénine dans Que faire?

\section{Trois voies}

En ce sens, dans Coriolan se profilent fondamentalement trois devenirs politiques potentiels, correspondant respectivement aux trois régimes archétypaux. Un devenir démocratique, pourvu que le peuple, assemblé en armes et mobilisé sous la houlette des tribuns, résiste à l'emprise du Sénat et parvienne à expulser Coriolan l'Arrogant comme fut chassé vingt ans plus tôt le dernier roi de Rome, Tarquin le Superbe. Un devenir aristocratique, pour peu que les sénateurs patriciens, après avoir subjugué la turbulente canaille, évincent le meilleur d'entre eux pour en briser les prétentions tyranniques. Un devenir monarchique, si Coriolan, appuyé sur des troupes fidèles par allégeance personnelle, parvient à en découdre avec la tourbe populaire et, selon les vœux de sa mère, à ranger les sénateurs sous sa coupe grâce à son mérite éclatant.

Historiquement, la République romaine pensa résoudre le conflit inexpiable entre ces trois formes de régime en octroyant à chacune d'elle une expression constitutionnelle dans la Cité, sur la base d'une répartition des pouvoirs. Au consul le pouvoir exécutif d'inspiration monarchique - certes scindé en deux, borné dans le temps et amputé des prérogatives trop absolues. Au sénat le pouvoir législatif, d'essence aristocratique, concentré entre les mains des patriciens. Au peuple, s'exprimant lors des comices et désormais 
représenté par des tribuns, le pouvoir démocratique, notamment celui d'élire leurs magistrats et au premier chef le premier d'entre eux, le consul. Mais ce volet démocratique était faussé par le classement inégalitaire en tribus, dont le vote pesait en fonction de la richesse.

Outre le conflit entre régimes, à lui seul chaque type de régime a tendance à dégénérer en sa forme maladive : la monarchie (pouvoir assumé par un seul homme) dégénère en tyrannie (pouvoir abusif d'un usurpateur), l'aristocratie (pouvoir confié aux meilleurs) en oligarchie (hégémonie ravie par une minorité illégitime), la démocratie (souveraineté effective du peuple citoyen) en démagogie (souveraineté fictive d'un peuple flatté et manipulé).

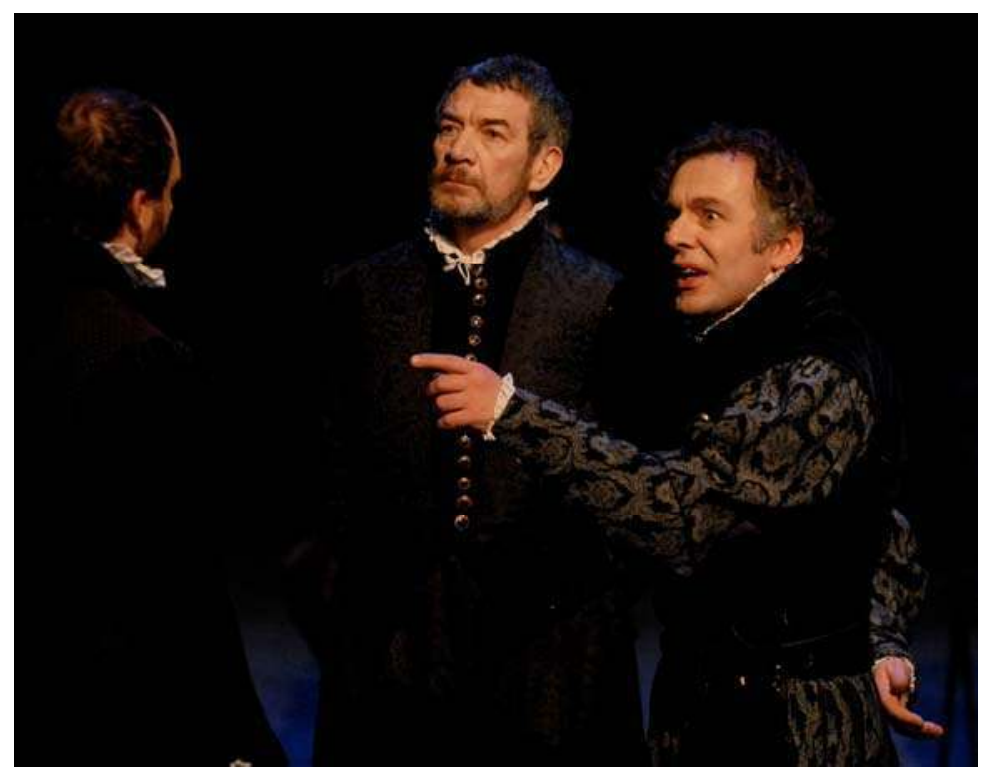

\section{L’impossible concordia}

Pour contrebalancer de tels risques, le régime mixte romain s'efforça d'instaurer une concordia: une harmonie entre les différentes composantes de la cité obtenue par la recherche du point médian entre ses aspirations contradictoires. Quatre siècles après la bataille de Corioles, cette quête éternelle d'un équilibre impossible sera encore 
celle d'un Cicéron se posant en dernier républicain et en homme de la synthèse, dans une République à l'agonie et une Rome écartelée par les guerres civiles ( $\mathrm{I}^{\mathrm{er}}$ siècle avant J.C.).

À cet égard, l'illustre orateur des derniers jours de la République se voit préfiguré, dans la République originelle de Coriolan, par le personnage de Ménénius, sénateur patricien modéré «ami du peuple», médiateur entre les factions populaire et

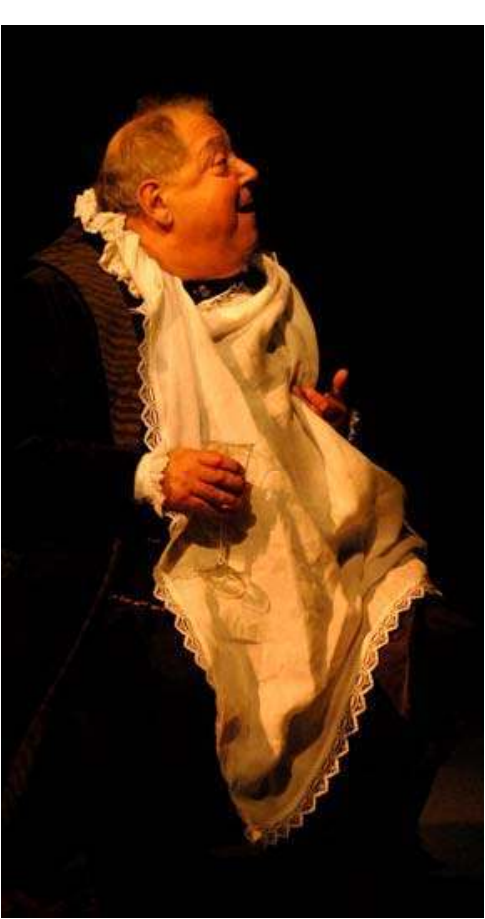
aristocratique, et surtout politicien rompu aux inévitables louvoiements, compromis et compromissions inhérents à la dynamique perpétuellement instable de la machine démocratique - puisque toute démocratie est, par nature, inachevée et inachevable, imparfaite et frustrante. Dès lors, l'enjeu est de savoir comment gérer, maîtriser, canaliser, détourner, sublimer cette irréductible et universelle frustration, afin que, même si nul dans la Cité n'est jamais pleinement satisfait, personne du moins n'en vienne au point d'exaspération qui le fera sortir du cadre démocratique pour se jeter dans la violence révolutionnaire.

\section{Entre deux maux}

Cette dialectique entre rage et tempérance court à travers toute la pièce, qui esquisse successivement les multiples configurations politiques envisageables. Au départ, une jacquerie spontanée se mue en mouvement plébéien révolutionnaire. Celui-ci tourne court sous le double effet d'une victoire démocratique effective (la concession par le 
Sénat à la plèbe d'une représentation populaire, avec la création du tribunat) et d'une défaite extérieure en perspective (l'anéantissement imminent de Rome par l'envahisseur volsque).

Pour sauver la patrie en danger, la Cité sonne la mobilisation générale et range tous ses citoyens, civils et militaires, sous la bannière de son meilleur soldat, Caius Martius. En renversant à la surprise collective le désastre annoncé en victoire héroïque et en prenant seul la ville de Corioles, le général Martius y gagne, avec la gloire, un surnom : Coriolan.

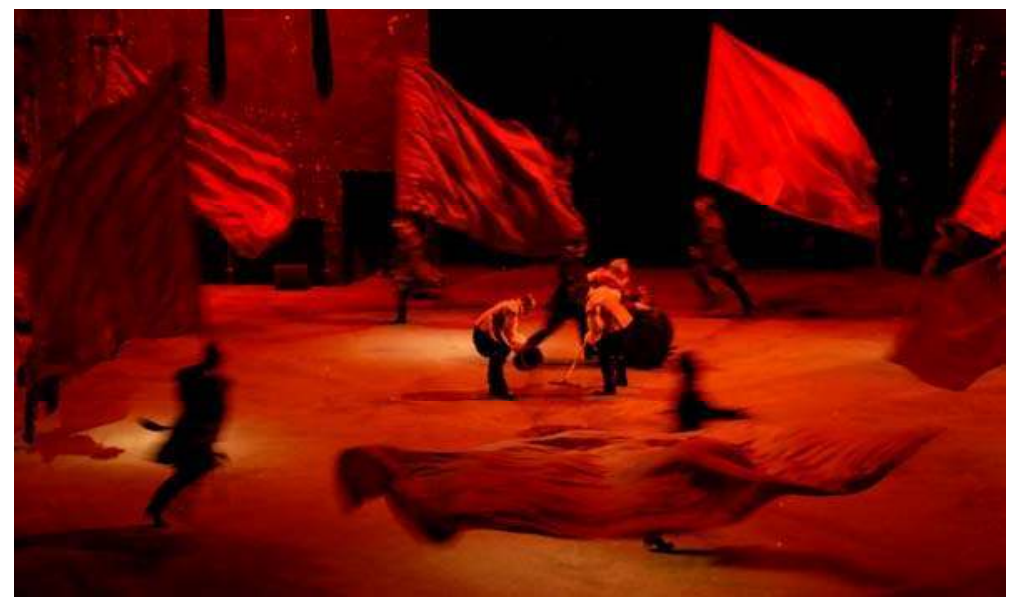

Mais le triomphe du général providentiel entraîne aussi un risque de césarisme (voire de bonapartisme) avant la lettre - soit l'exploitation de la faveur populaire pour capter le pouvoir à son profit exclusif, vider les institutions républicaines de leur substance et, finalement, se faire sacrer empereur. Qu'une telle conquête du pouvoir s'opère par un coup d'État militaire (menace qui pointe dès l'orée de la pièce), ou, pire, légalement, par les urnes, avec l'élection de Coriolan au consulat, elle s'annonce insupportable au parti populaire. Aussi, après débats, celui-ci décide-t-il d'invalider l'élection et de pousser à la faute le consul désigné. 


\section{La cause du peuple}

Sous les provocations des tribuns du peuple, excédé, l'intransigeant patricien se compromet en une incitation véhémente à l'écrasement de la plèbe voire en un appel à la guerre civile. Si bien que, contre toute attente, au nom de la Constitution violée, le parti populaire arrache le bannissement du grand homme auquel la patrie venait pourtant d'exprimer toute sa reconnaissance. Face au déchaînement des factions patriciennes et plébéiennes radicales, les tentatives de médiation politique, aussi bien militaire (le général et consul sortant Cominius) que civile (le sénateur Ménénius), n’ont pu qu'échouer.

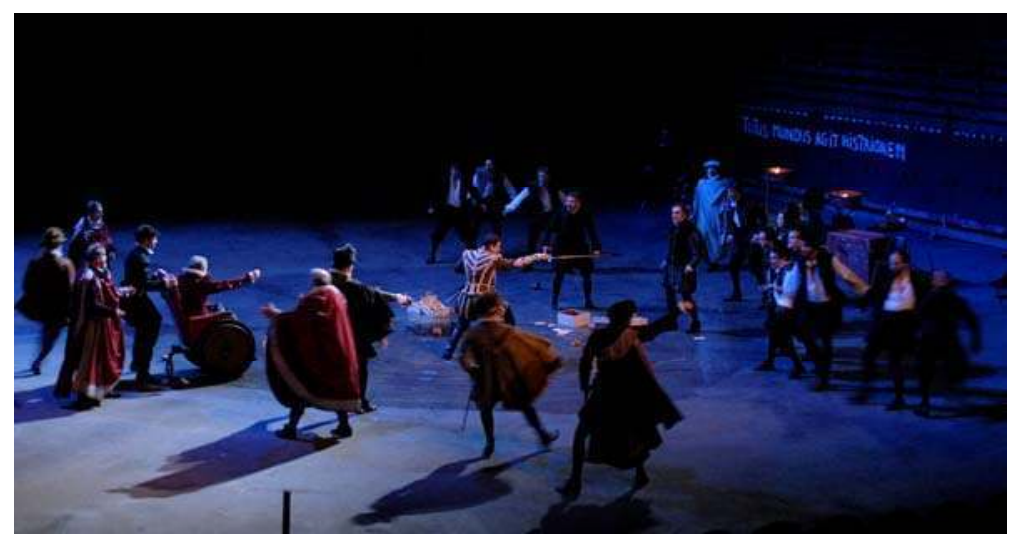

Ayant échappé à la monarchisation tant redoutée, Rome glisse alors vers une démocratie parlementaire qui ploie sous le poids de sa bureaucratisation et de sa routinisation. Ici comme ailleurs, tout ce qui a commencé en mystique finit en politique (Péguy) ${ }^{1}$. La faction populaire a déchu de mouvement militant en parti de gouvernement. Et l'aspiration à la révolution s'est réduite à l'exercice de la gestion. Avec le sénat pour siège du pouvoir, les tribuns de la plèbe comme maîtres à bord, et le petit peuple assagi réduit à manier l'encensoir, la machine politique tourne à vide, dans l'illusion d'une paix sociale.

Celle-ci a pourtant été achetée au prix fort par l'éviction du sauveur de la Cité, sacrifié sur l'autel de la concordia comme bouc émissaire. Sur son dos lacéré s'est opérée une réconciliation de façade

\footnotetext{
${ }^{1}$ Charle Péguy, Notre jeunesse, Gallimard, Folio Essai, 1993, p.115
} 
entre les mouvances démocratiques et aristocratiques modérées, peutêtre également soulagées d'avoir conjuré le danger monarchique. Seule n'a pas désarmé la branche aristocratique extrémiste conduite par la mère de Coriolan, Volumnia.

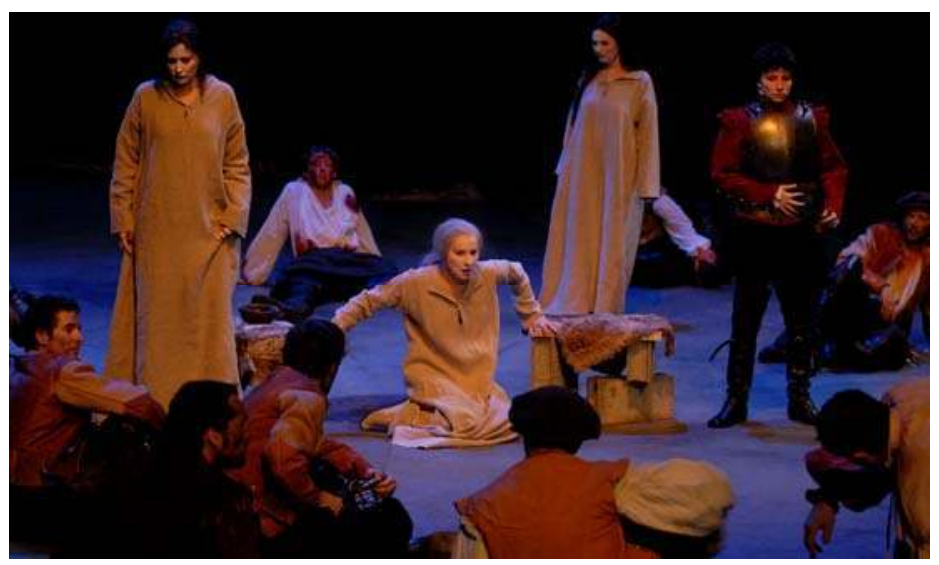

\section{Les chiens de guerre}

De ce ciel parlementaire sans nuages surgit la guerre éclair, lancée par un Coriolan vengeur et furieux. Passé tout entier à l'ennemi volsque avec armes et bagages, ou plutôt avec rage et drame, le héros patriote s'est changé en traître à la patrie, le général de la Louve en chien de guerre, le Romain exemplaire en ennemi à mort de Rome. Dans le camp d'en face, Coriolan a rejoint Aufidius, qu'il n'avait jusqu'alors de cesse de défier et de défaire en combat singulier, tel un frère ennemi ou un amant-rival. Combattant furieux et général impétueux tenu en suspicion par les sénateurs de son pays, à l'instar de Coriolan chez lui, Aufidius en est l'alter ego avorté, le double secondaire, le négatif à valeur de contrepoint - tel un reflet ondoyant dans l'eau sur lequel l'original aux traits incarnés aura toujours le dessus.

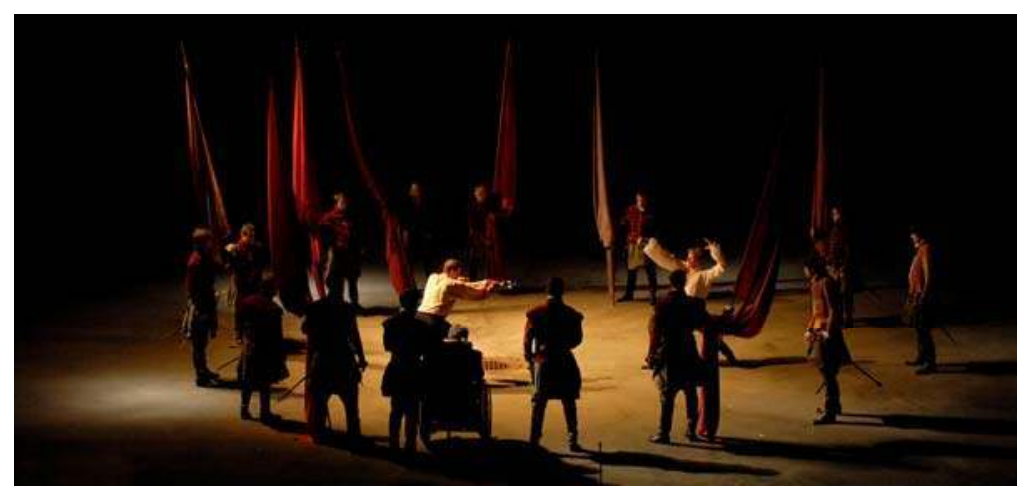


Après s'être affrontés comme d'autres s'aiment, sans répit et sans vergogne, ces deux êtres dits contraires révèlent leur complémentarité, leur symétrie, voire leur gémellité : ils entrent en fusion. À tour de rôle, chacun cannibalise l'autre. Aufidius cannibalise Coriolan en le faisant généralissime volsque objet de consommation de ses soldats, qui «le mettent dans leur bénédicité ${ }^{»}$. Coriolan cannibalise Audifius en usurpant sa place, en lui volant sa victoire et sa vengeance, et, par sa palinodie finale qui signera son retour chez les Romains, en trahissant sa trahison initiale. Et ainsi de suite. Envers centripète de la guerre civile centrifuge, cette fusion cannibale des

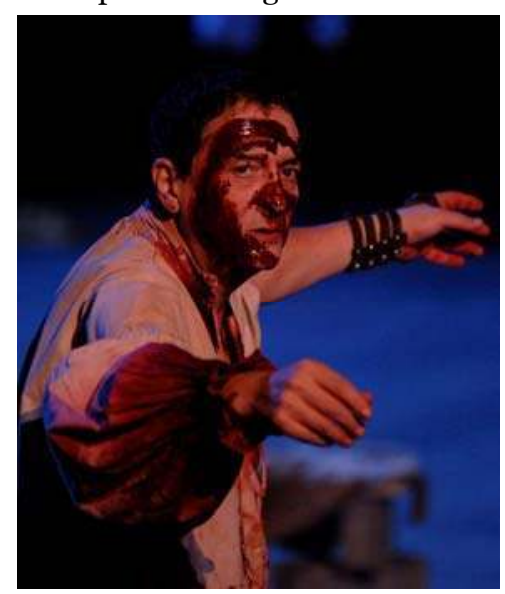
généraux prépare celle des deux peuples volsque et romain, qui seront in fine confondus en un seul et même populus romanus. Mais pour l'heure, tout à leur étreinte barbare, Aufidius et Coriolan ont franchi les bornes de la civilisation. Ils célèbrent leurs noces de sang par la guerre totale et la terreur - raids, massacres, razzias et terre brûlée -, tournant inlassablement autour de Rome en une voluptueuse danse de mort.

\section{La mère de toutes les batailles}

Rome mesure alors ce qu'elle a perdu : en bannissant son défenseur, elle s'est perdue elle-même. La menace de Coriolan résonne ici avec toute sa violence: "C'est moi qui vous bannis 3 !». Retour de boomerang, Martius éconduit sans ménagement les deux médiateurs jadis incapables d'empêcher son expulsion, Ménénius et Cominius, qui furent pourtant ses pères symboliques à la ville et à la guerre. Le temps des modérés et des modérateurs est passé.

\footnotetext{
${ }^{2}$ William Shakespeare, Coriolan, Iv.vii.3, Pléiade, Tragédies, tome II, trad. de J.-M. Déprats, p. 1291.

3 Ibid., III.iii.127, p. 1233.
} 


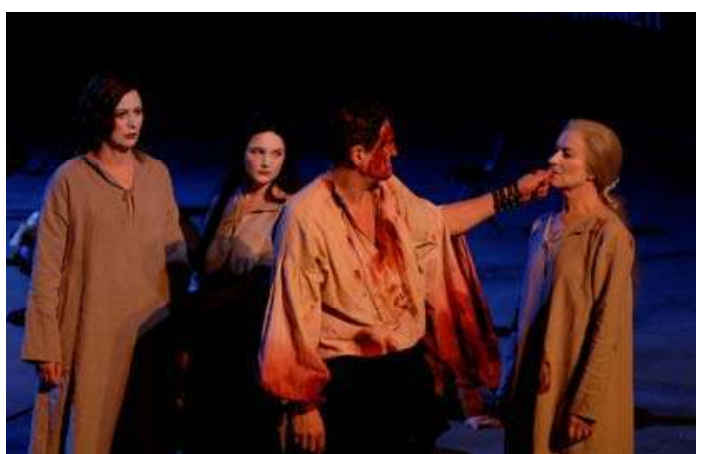

Seule la rhétorique universelle de sa mère (politique, affective, religieuse...) parvient à faire «fondre 4 » Coriolan, qui sur les injonctions maternelles renonce à la destruction de Rome pour se rallier au projet d'union des deux peuples. Lors de l'élection, Volumnia prêchait à son fils, rebelle à tout déguisement hormis comme ruse de guerre, les vertus du masque et de la comédie en politique - en vain. Par son triomphe, elle marque la victoire d'une conception machiavélienne, centralisatrice et impérialiste d'un pouvoir tenté par l'absolutisme sous des dehors conciliateurs. De fait, elle signifie que « la politique est la continuation de la guerre par d'autres moyens », selon la proposition de Foucault ${ }^{5}$ renversant l'assertion classique de Clausewitz ${ }^{6}$. Principe qu'à force de mépris le trop absolu Coriolan paie de sa vie, tandis que dans son sang se conjuguent les premières peuplades romaines, matrice d'où jaillira un empire millénaire.

Gérald GARUTTI

Théâtre National Populaire École Nationale Supérieure des Arts et Techniques du Théâtre Institut d'Etudes Politiques de Paris

\footnotetext{
${ }^{4}$ Ibid., v.iii.28, p. 1313 .

5 Michel Foucault, « La politique est la continuation de la guerre par d'autres moyens », in Dits et Écrits, Paris, Gallimard, tome II, p. 702-704.

${ }^{6}$ Carl von Clausewitz, De la guerre (1832).
} 